\title{
A Generalized Curvature of a Generalized Envelope
}

Tahir H. Ismail

tahir_hsis@uomosul.edu.iq

College of computers Sciences and Mathematics

University of Mosul

Received on: 27/6/2007
Ibrahim O. Hamad

ibrahim.hamad@su.edu.krd

College of Sciences

Salahaddin University

Accepted on: 4/11/2007

In this paper we study one of the applications of a generalized curvature [3] on the generalized envelope of a family of lines given in [7], [8], using some concepts of nonstandard analysis given by Robinson, A. [5] and axiomatized by Nelson, E..

Keywords: infinitesimals, monad, envelope, generalized curvature

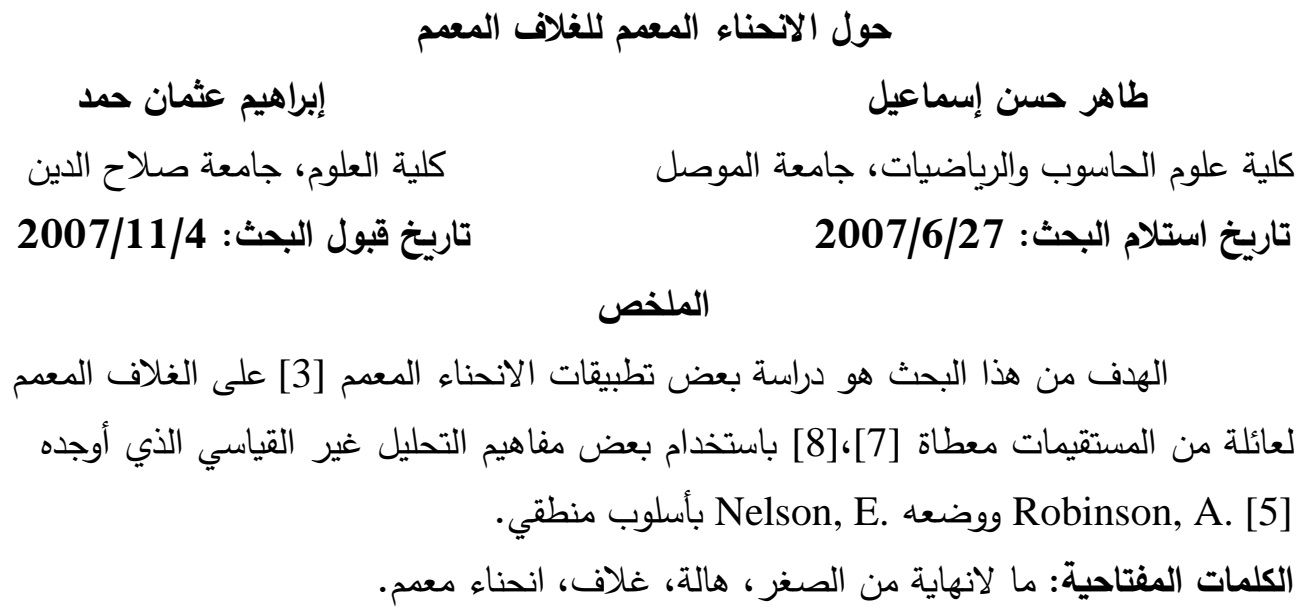

\section{1- Introduction:}

The following definitions and notations are needed throughout this paper.

Every concept concerning sets or elements defined in classical mathematics is called standard [4].

Any set or formula which does not involve new predicates "standard, infinitesimals, limited, unlimited...etc" is called internal, otherwise it is called external [2], [4].

A real number $\boldsymbol{x}$ is called unlimited if and only if $|\boldsymbol{x}|>\boldsymbol{r}$ for all positive standard real numbers, otherwise it is called limited [2].

A real number $\boldsymbol{x}$ is called infinitesimal if and only if $|\boldsymbol{x}|<\boldsymbol{r}$ for all positive standard real numbers $\boldsymbol{r}$ [2]. 
Two real numbers $x$ and $y$ are said to be infinitely close if and only if $\boldsymbol{x}-\boldsymbol{y}$ is infinitesimal and denoted by $\boldsymbol{x} \cong \boldsymbol{y}$ [2], [6].

If $\boldsymbol{x}$ is a limited number in $\mathbf{R}$, then it is infinitely close to a unique standard real number, this unique number is called the standard part of $\boldsymbol{x}$ or shadow of $\boldsymbol{x}$ denoted by $s t(x)$ or ${ }^{0} \boldsymbol{x}$ [2], [4].

If $\boldsymbol{x}$ is a real limited number, then the set of all numbers, which are infinitely close to $\boldsymbol{x}$, is called the monad of $\boldsymbol{x}$ and denoted by $\boldsymbol{m}(\boldsymbol{x})$ [2], [3]

A curve $\boldsymbol{v}$ is called envelope of a family of curves $\left\{\gamma_{\alpha}\right\}$ depending on a parameter $\boldsymbol{\alpha}$, if at each of its points, it is tangent to at least one curve of the family $\left\{\gamma_{\alpha}\right\}$, and if each of its segments is tangent to an infinite set of these curves [1].

The projective homogenous plane over $\mathbf{R}$, denoted by $\mathbf{P}_{\mathbf{R}}^{\mathbf{2}}$ is the set:

$\mathbf{P}_{\mathbf{R}}^{\mathbf{2}}=\mathbf{R}^{\mathbf{2}} \cup\{$ one point at $\infty$ for each equivalence classes of parallel lines $\}$, we denoted it by $(\boldsymbol{P H P})[1]$.

The projective homogeneous coordinates of a point $p(x, y) \in \mathbf{R}^{2}$ are $(x \boldsymbol{\alpha}, \boldsymbol{y} \boldsymbol{\alpha}, \boldsymbol{\alpha})$, where $\boldsymbol{\alpha}$ is any nonzero number, we denote it by $(\boldsymbol{P H C})$. In this sense the projective homogeneous coordinates of any point is not unique. [1]

By a parameterized differentiable curve, we mean a differentiable map $\gamma: \mathbf{I} \rightarrow \mathbf{R}^{\mathbf{3}}$ of an open interval $\mathbf{I}=(\boldsymbol{a}, \boldsymbol{b})$ of the real line $\mathbf{R}$ into $\square \mathbf{R}^{\mathbf{3}}$ such that: $\quad \gamma(t)=(x(t), y(t), z(t))=x(t) e_{1}+y(t) e_{2}+z(t) e_{3}$, and $x, y$, and $z$ are differentiable at $\boldsymbol{t}$; it is also called spherical curve [2].

\section{Definition 1.1 [7]}

Let $A=\gamma(t)$ be a standard point on the curve $\gamma$, then the following cases occur for the point $\boldsymbol{A}$ with the existence of the order of derivatives of $\gamma$ :

1- If $\gamma^{\prime} \neq 0, \gamma^{\prime \prime} \neq 0$ and $\gamma^{\prime} \cdot \gamma^{\prime \prime} \neq 0$ then the point is called biregular point.

2- If $\gamma^{\prime} \neq 0$ then the point is called regular point.

3- If $\gamma^{\prime} \neq 0$ and $\gamma^{\prime} \cdot \gamma^{\prime \prime} \neq 0$ then the point is called only regular point, and we say that the point is only regular point of order $p$-1 if $\gamma^{\prime} \neq 0$ and $\gamma^{\prime}=\gamma^{\prime \prime}=\cdots=\gamma^{(p-1)}=0$, but $\gamma^{\prime} \cdot \gamma^{(p)} \neq 0$. In this case we say that $p$ is the order of the first vector derivative not $\square$ collinear with $\gamma^{\prime}$

4- If $\gamma^{\prime}=0$ then the point is called singular point. In general if $\gamma^{\prime}=\gamma^{\prime \prime}=\cdots=\gamma^{(p-1)}=0$ but $\gamma^{(p)} \neq 0$, then the point is called singular point of order $\mathbf{p}$. 


\section{Theorem 1.2 [7]}

Let $\gamma$ be a standard curve of order $C^{n}$ and $\mathbf{A}$ be a standard singular point of order $p-1$ on $\gamma$; and let $B$ and $C$ be two points infinitely close to the point $A$, then the generalized curvature of $\gamma$ at the point denoted by $\boldsymbol{K}_{G}$ and given by

$$
K_{G}=\frac{(p !)^{\frac{q}{p}\left|x^{(p)} y^{(q)}-x^{(q)} y^{(p)}\right|}}{q !\left(x^{(p)^{2}}+y^{(p)^{2}}\right)^{\frac{q+p}{2 p}}}=\frac{(p !)^{\frac{q}{p}}\left|\gamma^{(p)} \times \gamma^{(q)}\right|}{q !\left\|\gamma^{(p)}\right\|^{\frac{q}{p}+1}},
$$

where $\boldsymbol{q}$ is the order of the first vector derivative of $\gamma$ not collinear with $\gamma^{(p)}$.

\section{Theorem 1.3 [7]}

If $\boldsymbol{p}_{\boldsymbol{k}}(t)=\boldsymbol{r}_{\boldsymbol{k}}(\mathrm{t})=\boldsymbol{q}_{\boldsymbol{k}}(\boldsymbol{t})=\mathbf{0}$ for $\boldsymbol{1} \leq \boldsymbol{k} \leq \boldsymbol{n}$ (n standard) and $\boldsymbol{p}_{\boldsymbol{n}}(\boldsymbol{t}), \boldsymbol{r}_{\boldsymbol{n}}(\boldsymbol{t}), \boldsymbol{q}_{\boldsymbol{n}}(\boldsymbol{t})$ are not all zeros, then the $\boldsymbol{P H C}$ points of $\gamma(\boldsymbol{t})$ are of the form $\left(\boldsymbol{p}_{n}(\boldsymbol{t}), \boldsymbol{r}_{n}(\boldsymbol{t})\right.$, $\left.\boldsymbol{q}_{\boldsymbol{n}}(\boldsymbol{t})\right)$ which does not depend on $\boldsymbol{e}$. Thus, we get the generalized nonclassical form of the envelope curve $\gamma(\boldsymbol{t})$ as follows:

$$
\begin{aligned}
(x(t), y(t)) & =\left(\frac{X_{e}(t)}{Z_{e}(t)}, \frac{Y_{e}(t)}{Z_{e}(t)}\right) \\
& =\left(\frac{v^{(n)}(t) w(t)-w^{(n)}(t) v(t)}{u^{(n)}(t) v(t)-v^{(n)}(t) u(t)}, \frac{w^{(n)}(t) u(t)-u^{(n)}(t) w(t)}{u^{(n)}(t) v(t)-v^{(n)}(t) u(t)}\right)
\end{aligned}
$$

\section{2- A Generalized Curvature of the Envelope of a Family of Lines}

Throughout this section, we give a curvature formula for the envelope of a family of lines $L_{t}: \boldsymbol{u}(t) \boldsymbol{x}+\boldsymbol{v}(t) \boldsymbol{y}+\boldsymbol{w}(t) z=0$ represented by the components $\boldsymbol{u}, \boldsymbol{v}$, and $\boldsymbol{w}$.

It is clear that every two infinitely closed points (points in the same monad) on the envelope curve of a family of lines determine two infinitely close lines in that monad.

That is, $\forall \boldsymbol{A}\left(\boldsymbol{t}_{\boldsymbol{o}}\right), \boldsymbol{B}\left(\boldsymbol{t}_{\boldsymbol{o}}\right) \in \gamma\left(\boldsymbol{t}_{\boldsymbol{o}}\right)$, where $\boldsymbol{B}\left(\boldsymbol{t}_{\boldsymbol{o}}+\alpha\right) \in \boldsymbol{m}\left(\boldsymbol{A}\left(\boldsymbol{t}_{\boldsymbol{o}}\right)\right)$ there exists a line $\boldsymbol{L}_{\boldsymbol{t}_{o}+\boldsymbol{a}} \in\left\{\boldsymbol{L}_{\boldsymbol{t}}\right\}$ such that $\boldsymbol{L}_{\boldsymbol{t}_{o}+\boldsymbol{a}}>\boldsymbol{L}_{\boldsymbol{t}_{o}}$ in $\boldsymbol{m}\left(\boldsymbol{A}\left(\boldsymbol{t}_{\boldsymbol{o}}\right)\right)$, where $\boldsymbol{m}\left(\boldsymbol{A}\left(\boldsymbol{t}_{\boldsymbol{o}}\right)\right)$ denotes the monad of the point $\boldsymbol{A}$, where $\alpha$ is an infinitesimal number.

For finding curvature formula of the envelope of a family of lines, we follow the following algorithm.

1. Find the envelope curve using Theorem $\mathbf{1 . 3}$ according to the case under consideration.

2. Find the singularity and collinearity order of the envelope curve.

3. Consider three infinitely closed points $\boldsymbol{A}\left(\boldsymbol{t}_{\boldsymbol{o}}\right), \boldsymbol{B}\left(\boldsymbol{t}_{\boldsymbol{o}}+\alpha\right)$ and $\boldsymbol{C}\left(\boldsymbol{t}_{\boldsymbol{o}}+\beta\right)$ on the envelope curve $g(t)$ such that 


$$
\boldsymbol{A}\left(\boldsymbol{t}_{\mathrm{o}}\right) \in \boldsymbol{L}_{\boldsymbol{t}_{o}}, \boldsymbol{B}\left(\boldsymbol{t}_{\mathrm{o}}+\alpha\right) \in \boldsymbol{L}_{\boldsymbol{t}_{o}+\boldsymbol{a}} \text { and } \boldsymbol{C}\left(\boldsymbol{t}_{\mathrm{o}}+\beta\right) \in \boldsymbol{L}_{\boldsymbol{t}_{o}+\boldsymbol{b}}
$$

4. Apply the generalized curvature formula given in Theorem $\mathbf{1 . 2}$ at the points $\boldsymbol{A}\left(\boldsymbol{t}_{\boldsymbol{o}}\right), \boldsymbol{B}\left(\boldsymbol{t}_{\boldsymbol{o}}+\alpha\right)$ and $\boldsymbol{C}\left(\boldsymbol{t}_{\boldsymbol{o}}+\beta\right)$.

Where $\alpha$ and $\beta$ are infinitesimal numbers.

The following theorems will give a new formula of the generalized curvature of the envelope of a family of lines.

\section{Theorem 2.1}

Let $\boldsymbol{A}=\left(\boldsymbol{t}_{\boldsymbol{o}}\right)$ be a regular point of the envelope curve $\gamma$ of the family $L_{t}: \boldsymbol{u}(\boldsymbol{t}) \boldsymbol{X}+\boldsymbol{v}(\boldsymbol{t}) \boldsymbol{Y}+\boldsymbol{w}(t) \boldsymbol{Z}=\boldsymbol{O}$ in $\boldsymbol{P H C}$, then the generalized curvature $\boldsymbol{K}_{G}$ of the envelope curve at a point $\boldsymbol{A}$ is given by

$$
\frac{\left|\left(r^{\prime}(t) q^{\prime \prime}(t)-r^{\prime \prime}(t) q^{\prime}(t)\right)^{2}+\left(p^{\prime \prime}(t) q^{\prime}(t)-p^{\prime}(t) q^{\prime \prime}(t)\right)^{2}+\left(p^{\prime}(t) r^{\prime \prime}(t)-p^{\prime \prime}(t) r^{\prime}(t)\right)^{2}\right|^{\frac{1}{2}}}{2\left|p^{\prime}(t)^{2}+q^{\prime}(t)^{2}+r^{\prime}(t)^{2}\right|^{\frac{3}{2}}},
$$

where $\boldsymbol{p}(\boldsymbol{t}), \boldsymbol{r}(\boldsymbol{t})$ and $\boldsymbol{q}(\boldsymbol{t})$ are as given in Theorem 1.3 for $\boldsymbol{n}=\boldsymbol{1}$

\section{Proof:}

Let $A=\gamma\left(t_{o}\right)$ be a standard point on the envelope of the curve $\gamma$, and $\boldsymbol{B}=\gamma\left(\boldsymbol{t}_{\boldsymbol{o}}+\alpha\right), \boldsymbol{C}=\gamma\left(\boldsymbol{t}_{\boldsymbol{o}}+\beta\right)$ be two points infinitely close to $\boldsymbol{A}$. Let $\boldsymbol{L}_{\boldsymbol{t}}, \boldsymbol{L}_{\boldsymbol{t}+\boldsymbol{a}}$ and $\boldsymbol{L}_{t+b}$ be three lines of the family $\left\{\boldsymbol{L}_{t}\right\}$ having $\boldsymbol{A}, \boldsymbol{B}$ and $\boldsymbol{C}$ as contact points with the envelope curve, respectively.

Then;

$$
\begin{aligned}
& L_{t}: u(t) X+v(t) Y+w(t) Z=0, \\
& L_{t+a}: u(t+\varepsilon) X+v(t+\square \varepsilon) Y+w(t+\square \varepsilon) Z=0, \\
& L_{t+b}: u(t+\varepsilon) X+v(t+\square \varepsilon) Y+w(t+\square \varepsilon) Z=0 .
\end{aligned}
$$

Since, the point $\boldsymbol{A}$ is regular, then Theorem $\mathbf{1 . 3}$ for $\boldsymbol{n}=\boldsymbol{1}$ is satisfied, and therefore $\gamma(t)=\left(p_{1}(t), r_{I}(t), q_{1}(t)\right)$

Using the spherical case of the generalized curvature given in Theorem 1.2 for a curve $\gamma=(x(t), y(t), z(t))$, we get

$$
K_{G}=\frac{\left|\left(y^{\prime} z^{\prime \prime}-y^{\prime \prime} z^{\prime}\right)^{2}+\left(x^{\prime \prime} z^{\prime}-x^{\prime} z^{\prime \prime}\right)^{2}+\left(x^{\prime} y^{\prime \prime}-x^{\prime \prime} y^{\prime}\right)^{2}\right|^{\frac{1}{2}}}{2\left|x^{\prime 2}+y^{\prime 2}+z^{\prime 2}\right|^{\frac{3}{2}}}
$$

Now replacing each of $\boldsymbol{x}, \boldsymbol{y}$ and $\boldsymbol{z}$ by $\boldsymbol{p}_{1}(\boldsymbol{t}), \boldsymbol{r}_{I}(\mathrm{t})$ and $\boldsymbol{q}_{\boldsymbol{I}}(\boldsymbol{t})$, respectively, we get the required result.

\section{Theorem 2.2}


Let $\boldsymbol{A}=\gamma\left(\boldsymbol{t}_{\boldsymbol{o}}\right)$ be a singular point of the envelope curve $\gamma$ of order $\boldsymbol{n - 1}$, and let $\boldsymbol{m}$ be the order of the first nonzero derivative which is not collinear with $\gamma^{(n)}(\boldsymbol{t})$, that is, $\gamma^{\prime}(\boldsymbol{t})=\gamma^{\prime \prime}(\boldsymbol{t})=\cdots=\gamma^{(\boldsymbol{n}-\boldsymbol{I})}(\boldsymbol{t})=\mathbf{0}, \gamma^{(\boldsymbol{n})}(\boldsymbol{t}) \neq \boldsymbol{0}$, and $\gamma^{\prime}(t) \cdot \gamma^{\prime \prime}(t)=\gamma^{\prime}(t) \square \cdot \gamma^{\prime \prime \prime}(t)=\cdots=\square \gamma^{\prime}(t) \square \cdot \gamma^{(m-1)}(t)=\cdots=\gamma^{(n-1)} \square .(t) \gamma^{(m-1)}(t)=0$, $\gamma^{(n)}(t) \cdot \gamma^{(m)}(t) \neq 0$

Then, the generalized curvature $\boldsymbol{K}_{\boldsymbol{G}}$ of the envelope curve $\gamma$ at the points of the monad of $\boldsymbol{A}$ is given by

$$
\frac{(\boldsymbol{n} !)^{\frac{m}{n}}\left|\left(\boldsymbol{r}^{(n)} \boldsymbol{q}^{(m)}-\boldsymbol{r}^{(m)} \boldsymbol{q}^{(n)}\right)^{2}+\left(\boldsymbol{p}^{(m)} \boldsymbol{q}^{(n)}-\boldsymbol{p}^{(n)} \boldsymbol{q}^{(m)}\right)^{2}+\left(\boldsymbol{p}^{(n)} \boldsymbol{r}^{(m)}-\boldsymbol{p}^{(m)} \boldsymbol{r}^{(n)}\right)^{2}\right|^{\frac{1}{2}}}{\boldsymbol{m} !\left|\boldsymbol{p}^{(n)^{2}}+\boldsymbol{q}^{(n)^{2}}+\boldsymbol{r}^{(n)^{2}}\right|^{\frac{m+n}{2 n}}}
$$

Moreover, the Cartesian coordinate of the generalized curvature $\boldsymbol{K}_{\boldsymbol{G}}$ of the envelope curve $\gamma$ at the points of the monad of $\boldsymbol{A}$ is given by

$$
\frac{(n !)^{\frac{m}{n}}\left|\left(\frac{p(t)}{q(t)}\right)^{(n)}\left(\frac{\boldsymbol{r}(t)}{q(t)}\right)^{(m)}-\left(\frac{\boldsymbol{p}(t)}{\boldsymbol{q}(t)}\right)^{(m)}\left(\frac{\boldsymbol{r}(t)}{\boldsymbol{q}(t)}\right)^{(n)}\right|}{m !\left|\left(\frac{\boldsymbol{p}(\boldsymbol{t})}{\boldsymbol{q}(\boldsymbol{t})}\right)^{(n)^{2}}+\left(\frac{\boldsymbol{r}(\boldsymbol{t})}{\boldsymbol{q}(\boldsymbol{t})}\right)^{(n)^{2}}\right|^{\frac{m+n}{2 n}}}
$$

where $n$ and $m$ are positive integer numbers.

\section{Proof:}

First, applying the spherical case of the generalized curvature given in Theorem 1.2 at $\boldsymbol{x}=p_{1}(t), \boldsymbol{y}=\boldsymbol{r}_{1}(t)$ and $\boldsymbol{z}=\boldsymbol{q}_{1}(t)$, we get the generalize curvature formula (2.2.1). Since the point $\left(p_{I}(t), r_{I}(t), q_{I}(t)\right)$ in $P H C$ is equivalent to the point $\left(\boldsymbol{p}_{1}(\boldsymbol{t}) / \boldsymbol{q}_{1}(t), \boldsymbol{r}_{1}(\boldsymbol{t}) / \boldsymbol{q}_{1}(t), \boldsymbol{l}\right)$, so again, applying the spherical case of generalized curvature, we get

$$
K_{G}=\frac{(n !)^{\frac{m}{n}}\left|\left(y^{(n)} z^{(m)}-y^{(m)} z^{(n)}\right)^{2}+\left(z^{(n)} x^{(m)}-z^{(m)} x^{(n)}\right)^{2}+\left(x^{(n)} y^{(m)}-x^{(m)} y^{(n)}\right)^{2}\right|^{\frac{1}{2}}}{m !\left|x^{(n)^{2}}+y^{(n)^{2}}+z^{(n)^{2}}\right|^{\frac{m+n}{2 n}}} \ldots
$$

Thus, putting $\boldsymbol{x}=p_{1}(\boldsymbol{t}) / q_{1}(\mathrm{t}), \boldsymbol{y}=\boldsymbol{r}_{1}(\boldsymbol{t}) / \boldsymbol{q}_{1}(\boldsymbol{t})$ and $\boldsymbol{z}=\boldsymbol{1}$, in (2.2.3), we obtain the formula (2.2.2).

\section{Corollary 2.3}

Let $\boldsymbol{A}=\gamma\left(\boldsymbol{t}_{\boldsymbol{o}}\right)$ be a singular point of the envelope curve $\gamma$ satisfying the hypothesis of Theorem 2.2.

Moreover, let the coefficient vector $(\boldsymbol{u}(\boldsymbol{t}), \boldsymbol{v}(\boldsymbol{t}), \boldsymbol{w}(\boldsymbol{t}))$ of the envelope curve has a singularity of order $\boldsymbol{n - 1}$, then the generalized curvature $\boldsymbol{K}_{\boldsymbol{G}}$ of the envelope curve $\gamma$ at points in the monad of $\boldsymbol{A}$ is given by 
$\frac{(n !)^{\frac{m}{n}}\left|\left(\boldsymbol{r}_{n}(t) \boldsymbol{q}_{m}(t)-\boldsymbol{r}_{m}(t) \boldsymbol{q}_{n}(t)\right)^{2}+\left(\boldsymbol{p}_{m}(t) \boldsymbol{q}_{n}(t)-\boldsymbol{p}_{n}(t) \boldsymbol{q}_{m}(t)\right)^{2}+\left(\boldsymbol{p}_{n}(t) \boldsymbol{r}_{m}(t)-\boldsymbol{p}_{m}(t) \boldsymbol{r}_{n}(t)\right)^{2}\right|^{\frac{1}{2}}}{m !\left|\boldsymbol{p}_{n}^{2}(t)+\boldsymbol{q}_{n}^{2}(t)+\boldsymbol{r}_{n}^{2}(t)\right|^{\frac{m+n}{2 n}}}$

and the cartesian coordinate curvature $\boldsymbol{K}_{G}(\boldsymbol{t})$ of the envelope

$$
\frac{\begin{array}{c}
\text { curve } \gamma \text { at } \boldsymbol{A} \text { is given by } \\
(\boldsymbol{n} !)^{\frac{m}{n}}\left|\left(\frac{\boldsymbol{p}_{n}(t)}{\boldsymbol{q}_{n}(t)}\right) \cdot\left(\frac{\boldsymbol{r}_{m}(t)}{\boldsymbol{q}_{m}(t)}\right)-\left(\frac{\boldsymbol{p}_{m}(t)}{\boldsymbol{q}_{m}(t)}\right) \cdot\left(\frac{\boldsymbol{r}_{n}(t)}{\boldsymbol{q}_{n}(t)}\right)\right|
\end{array}}{m !\left|\left(\frac{\boldsymbol{p}_{n}(t)}{\boldsymbol{q}_{n}(t)}\right)^{2}+\left(\frac{\boldsymbol{r}_{n}(t)}{\boldsymbol{q}_{n}(t)}\right)^{2}\right|^{\frac{m+n}{2 n}}}
$$

\section{Proof:}

By Theorem 2.2 we have

$$
\boldsymbol{K}_{G}(\boldsymbol{t})=\frac{(\boldsymbol{n} !)^{\frac{m}{n}}\left|\left(\boldsymbol{r}^{(n)} \boldsymbol{q}^{(m)}-\boldsymbol{r}^{(m)} \boldsymbol{q}^{(n)}\right)^{2}+\left(\boldsymbol{p}^{(m)} \boldsymbol{q}^{(n)}-\boldsymbol{p}^{(n)} \boldsymbol{q}^{(m)}\right)^{2}+\left(\boldsymbol{p}^{(n)} \boldsymbol{r}^{(m)}-\boldsymbol{p}^{(m)} \boldsymbol{r}^{(n)}\right)^{2}\right|^{\frac{1}{2}}}{\boldsymbol{m} !\left|\boldsymbol{p}^{(n)^{2}}+\boldsymbol{q}^{(n)^{2}}+\boldsymbol{r}^{(n)^{2}}\right|^{\frac{m+n}{2 n}}}
$$

Since the coefficient vector $(\boldsymbol{u}(t), \boldsymbol{v}(\boldsymbol{t}), \boldsymbol{w}(\boldsymbol{t}))$ of the envelope curve has a singularity of order $\boldsymbol{n}$-1, so we get

$$
u^{\prime}(t)=v^{\prime}(t)=w^{\prime}(t)=\cdots=u^{(n-1)}(t)=v^{(n-1)}(t)=w^{(n-1)}(t)=0,
$$

and $\quad\left(u^{(n)}(t), v^{(n)}(t), w^{(n)}(t)\right) \neq 0$

Therefore,

$$
\begin{array}{r}
p^{(n)}(t)=v^{(n)}(t) w(t)-w^{(n)}(t) v(t)=p_{n}(t) \\
r^{(n)}(t)=w^{(n)}(t) u(t)-u^{(n)}(t) w(t)=r_{n}(t) \\
q^{(n)}(t)=u^{(n)}(t) v(t)-v^{(n)}(t) u(t)=q_{n}(t)
\end{array}
$$

Hence, the result of the first part is proved.

To prove the second part put $\boldsymbol{x}=\mathbf{p}_{\mathbf{n}}(\mathbf{t}) / \mathbf{q}_{\mathbf{n}}(\mathbf{t}), \boldsymbol{y}=\mathbf{r n}_{\mathbf{n}}(\mathbf{t}) / \mathbf{q}_{\mathbf{n}}(\mathbf{t})$ and $\boldsymbol{z}=\mathbf{1}$ and then apply the spherical curvature formula (2.2.3) to obtain the formula (2.3.2).

\section{Corollary 2.4}

Let $\boldsymbol{A}=\gamma\left(\boldsymbol{t}_{\boldsymbol{o}}\right)$ be a singular point of the envelope curve $\gamma$ satisfying the hypothesis of Theorem 2.2. Let $\square \gamma(t)=(p(t), r(t), q(t))$ be such that $q(t)$ has a nonzero constant value, then the generalized curvature $\boldsymbol{K}_{G}$ of the envelope curve $\gamma$ at points of the monad of $\boldsymbol{A}$ is given by

$$
\frac{(n !)^{\frac{m}{n}}\left|\left(p^{(n)}(t) r^{(m)}(t)-p^{(m)}(t) r^{(n)}(t)\right)^{2}\right|}{m !\left|p^{2}(t)+r^{2}(t)\right|^{\frac{m+n}{2 n}}} \cdot q^{\frac{m-n}{n}}
$$


Proof:

Without loss of generality we use the cartesian coordinate form (2.2.2) of Theorem 2.2 to obtain

$$
K_{G}(t)=\frac{(n !)^{\frac{m}{n}}\left|\left(\frac{p(t)}{q(t)}\right)^{(n)}\left(\frac{\boldsymbol{r}(t)}{q(t)}\right)^{(m)}-\left(\frac{p(t)}{q(t)}\right)^{(m)}\left(\frac{\boldsymbol{r}(t)}{q(t)}\right)^{(n)}\right|}{m !\left|\left(\frac{p(t)}{q(t)}\right)^{(n)^{2}}+\left(\frac{\boldsymbol{r}(t)}{q(t)}\right)^{(n)^{2}}\right|^{\frac{m+n}{2 n}}}
$$

Since the value of $\boldsymbol{q}(\boldsymbol{t})$ is constant, we get

$$
\begin{aligned}
& K_{G}(t)=\frac{(n !)^{\frac{m}{n}}\left(\frac{\boldsymbol{I}}{q}\right)^{2}\left|p(t)^{(n)} r(t)^{(m)}-p(t)^{(m)} r(t)^{(n)}\right|}{m !\left(\frac{\mathbb{1}}{q}\right)^{\frac{m+n}{n}}\left|p(t)^{(n)^{2}}+r(t)^{(n)^{2}}\right|^{\frac{m+n}{2 n}}} \\
& =\frac{(n !)^{\frac{m}{n}}\left|\left(p^{(n)}(t) r^{(m)}(t)-p^{(m)}(t) r^{(n)}(t)\right)^{2}\right|}{m !\left|p^{2}(t)+r^{2}(t)\right|^{\frac{m+n}{2 n}}} \cdot q^{\frac{m-n}{n}} .
\end{aligned}
$$

\section{Remark 2.5}

If $q(t)=0$ then, by using either equation (2.2.1) or the equation (2.3.1), we can find a spherical generalized curvature $\boldsymbol{K}_{G}$, but it does not represent a real curvature of the envelope curve. We shall call such value of curvature Ideal Curvature of a curve $\gamma$ at points of the monad of $\boldsymbol{A}=\gamma\left(\boldsymbol{t}_{\boldsymbol{o}}\right)$.

\section{Example 2.6}

Consider the family of lines $2 x-3 t y+t^{3}=0$

By applying the algorithm given at the beginning of this section, we get

$$
\begin{array}{llll}
u=2 & u^{\prime}=0 & u^{\prime \prime}=0 & u^{\prime \prime \prime}=0 \\
v=-3 t & v^{\prime}=-3 & v^{\prime \prime}=0 & v^{\prime \prime \prime}=0 \\
w=2 t^{3} & w^{\prime}=6 t^{2} & w^{\prime \prime}=12 t & w^{\prime \prime \prime}=12
\end{array}
$$

Now we determine the singularity and collinearity

$$
\gamma(0)=(0,0) \quad \gamma^{\prime}(0)=(0,0) \quad \gamma^{\prime \prime}(0)=(0,2) \quad \gamma^{\prime \prime \prime}(0)=(12,0)
$$

Thus $\gamma$ has a first singularity order (that is $\boldsymbol{n}=\mathbf{2}$ ) and the order of collinearity is equal to 3 . The envelope curve $\gamma(t)$ is given by $(X \varepsilon(t), Y \varepsilon(t), Z \varepsilon(t))$

$$
\begin{aligned}
& =\left(v^{\prime}(t) w(t)-w^{\prime}(t) v(t), w^{\prime}(t) u(t)-u^{\prime}(t) w(t), u^{\prime}(t) v(t)-v^{\prime}(t) u(t)\right) \\
& =\left(6 t^{3}, 6 t^{2}, 12\right)
\end{aligned}
$$


Since the value of $\mathbf{q}(\mathbf{t})$ is constant, so using Corollary 2.4, we get,

$$
K_{G}=\frac{(2 !)^{\frac{3}{2}}\left|\left(p^{(2)}(t) r^{(3)}(t)-p^{(3)}(t) r^{(2)}(t)\right)^{2}\right|}{3 !\left|p^{2}(t)+r^{2}(t)\right|^{\frac{3+2}{2 \times 2}}} \cdot q^{\frac{3-2}{2}}=\frac{1}{\sqrt{6}} \cdot \sqrt{12}=\sqrt{2}
$$

Note that if we use the cartesian coordinate, we find that $\gamma(t)$ is equal to

$$
\begin{aligned}
(x(t), y(t)) & =\left(\frac{X_{e}(t)}{Z_{e}(t)}, \frac{Y_{e}(t)}{Z_{e}(t)}\right)=\left(\frac{v^{\prime}(t) w(t)-w^{\prime}(t) v(t)}{u^{\prime}(t) v(t)-v^{\prime}(t) u(t)}, \frac{w^{\prime}(t) u(t)-u^{\prime}(t) w(t)}{u^{\prime}(t) v(t)-v^{\prime}(t) u(t)}\right) \\
& =(1 / 2)\left(t^{3}, t^{2}\right)
\end{aligned}
$$

Here $\gamma$ also has a first singularity order (that is $\boldsymbol{n = 2}$ ) and the order of collinearity is equal to 3 . Thus by using the usual two dimensional forms of the generalized curvature, we get, (see Figure 2.3 )

$$
K_{G}=\frac{(2 !)^{\frac{3}{2}}\left|\left(x^{(2)}(t) y^{(3)}(t)-x^{(3)}(t) y^{(2)}(t)\right)^{2}\right|}{3 !\left|\left(x^{(2)}(t)\right)^{2}+\left(y^{(2)}(t)\right)^{2}\right|^{3+2}}=\frac{(2 !)^{\frac{3}{2}}|3 t \cdot 0-3 \cdot 1|}{\left.3 !\left|(3 t)^{2}+(1)^{2}\right|^{3+2}\right|_{t=0} ^{2 \times 2}}=\sqrt{2}
$$

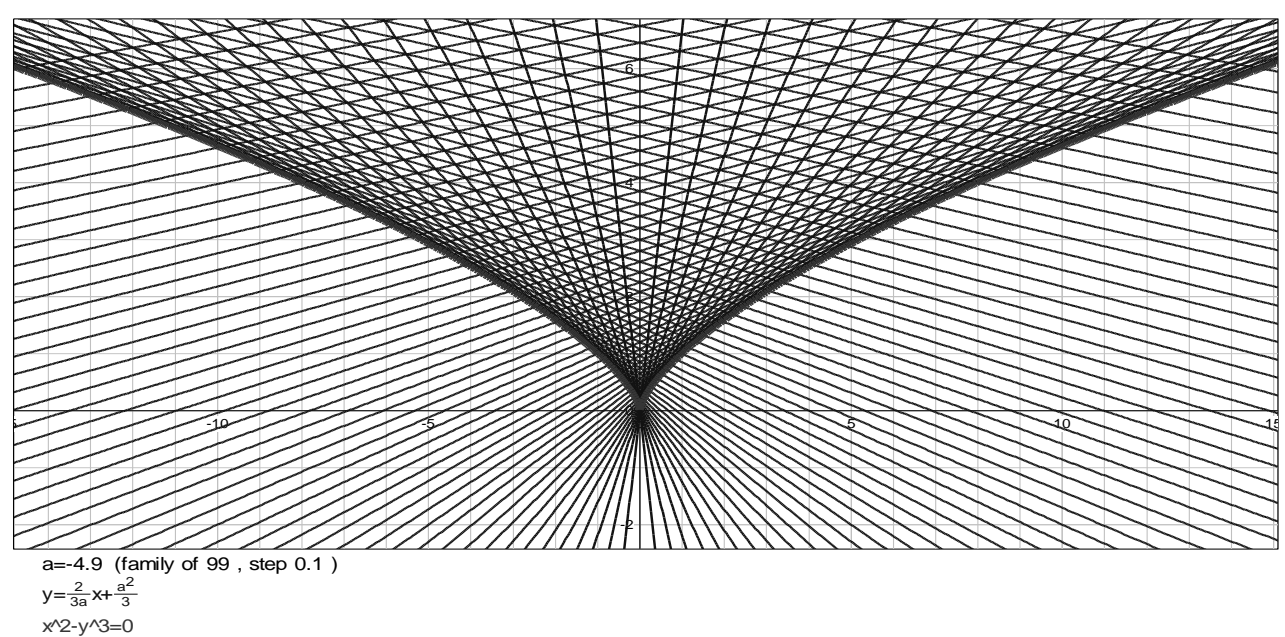

Figure 2.3

Remark: The graph of the equation of the above example is plotted with specific software Omnigraph V3.1b-2005. 


\section{REFERENCES}

[1] Carmo, M. (1976); Differential Geometry of Curves and Surfaces, Prentice-Hall, INC, New York.

[2] Diener, F.\& Diener M. (1996); Nonstandard Analysis in Practice, Springer-Verlag, Berlin,HeildeBerg.

[3] Keisler, H. J. (2005); Elementary Calculus-2ed-An Infinitesimal Approach, Creative Commons, 559 Nathan Abbott, Stanford, California, 93405, USA.

[4] Nelson, E. (1977); Internal set Theory-A New Approach to Nonstandard Analysis, Bull. Amer. Math. Soc, Vol.83, No.6, pp.1165-1198.

[5] Robinson, A. (1974); Nonstandard Analysis $2^{\text {ED }}$, North-Holland Pub.Comp.

[6] Rosinger, E. E(2004); Short Introduction to Nonstandard Analysis, arXiv: math. GM/ 0407178 v1 10 Jul.

[7] Tahir H. Ismail \& Ibrahim O. Hamad(2006); On Generalized Curvature, Raf. J. Comp. Sc. and Maths., Vol. 3, No.2, pp.83-98.

[8] Tahir H. Ismail \& Ibrahim O. Hamad; A Nonstandard Generalization of Envelopes, To Appear 